\title{
The impact of adherence and disease control on resource use and charges in patients with mild asthma managed on inhaled corticosteroid agents
}

This article was published in the following Dove Press journal:

Patient Preference and Adherence

24 June 2010

Number of times this article has been viewed

\section{P Navaratnam ${ }^{\prime}$ \\ HS Friedman ${ }^{2}$ \\ E Urdaneta ${ }^{3}$}

'Eympres Research, LLC, Hilliard, $\mathrm{OH}$, USA; ${ }^{2}$ Analytic Solutions, LLC, New York, NY, USA; ${ }^{3}$ Schering-Plough Corporation, Kenilworth, NJ, USA
Correspondence: Prakash Navaratnam Eympres Research, LLC, 4660 Stonehill St, Hilliard, OH 43026, USA

$\mathrm{Tel}+\mathrm{I}(6 \mid 4) 57 \mid-7299$

Fax +I (6I4) 57I-7299

Email Eympres@gmail.com
Objective: Inadequate asthma control may affect asthma resource use and treatment charges, consequently contributing to the growing economic burden of asthma. The study objective was to determine the impact of medication adherence and asthma control on resource use and charges in mild asthmatic patients treated with inhaled corticosteroids (ICSs).

Research design and methods: A claims database was analyzed retrospectively from October 2001-December 2007 to identify mild asthmatic patients aged 12-65 years who began ICS treatment. Demographics, drug utilization, and resource use for each patient were identified for the 365-day period before and after the index date (pre-index and post-index periods, respectively). Patients were designated as having high control high adherence (HCHA) or low control low adherence (LCLA) based on post-index exacerbations and the percentage of days covered; not all patients who qualified for study inclusion met adherence designation requirements. Differences between the HCHA and LCLA cohorts in resource use (eg, asthma treatment days) and asthma-related treatment charges were assessed.

Results: Compared with the HCHA cohort $(n=483)$, the LCLA cohort $(n=258)$ had more asthma treatment days ( 2.9 vs 3.9, respectively; $P<0.0001)$ and higher overall asthma treatment charges ( $\$ 2655$ vs $\$ 3345$, respectively; $P<0.0001$ ) in the post-index period. An adjusted odds ratio suggested that patients receiving mometasone furoate (MF) were approximately 5 times more likely to belong to the HCHA cohort than patients receiving any other ICS $(P<0.0001)$.

Conclusions: Better asthma control and adherence to prescribed ICSs are associated with lower asthma-related resource use and charges. Mild asthmatic patients receiving MF were more likely to be in the HCHA cohort than patients receiving other ICSs, perhaps due to the once-daily dosing of MF. Current NAEPP guidelines recommend low-dose ICS monotherapy for mild persistent asthma; thus, it is critical to optimize mild persistent asthma control and limit unnecessary resource use and charges.

Keywords: adherence, asthma control, beclomethasone dipropionate, budesonide, fluticasone propionate, mometasone furoate, retrospective claims analysis

\section{Introduction}

The goal of asthma treatment is to achieve and maintain adequate asthma control, which can be measured by assessing the severity of asthma symptoms, the incidence of asthma-related exacerbations, and patient quality of life. ${ }^{1}$ Current National Asthma Education and Prevention Program (NAEPP) guidelines define asthma control as reduced impairment (eg, prevention of chronic asthma symptoms and infrequent $[\leq 2$ days per week] use of short-acting $\beta_{2}$-agonist [SABA] rescue medication) and reduced risk (eg, prevention of recurrent asthma exacerbations and minimization of the need for 
emergency department [ED] visits). ${ }^{1}$ Inadequate asthma control may directly affect asthma resource use and treatment charges, which has the potential to significantly affect the growing global economic burden of asthma. ${ }^{2}$ For example, uncontrolled asthma leads to increased use of SABAs and oral corticosteroids (OCSs) as well as increased ED, hospital, and outpatient visits, ${ }^{3,4}$ and has been shown to correlate with higher asthma-related direct medical expenditures and indirect charges. ${ }^{3}$ In particular, the cost of rescue SABA therapy has recently increased because of new US Food and Drug Administration regulations that eliminate the use of ozone-depleting chlorofluorocarbon (CFC) propellants in all metered-dose inhalers (MDIs). ${ }^{5}$ Although this new regulation is environmentally important, it has resulted in the replacement of generic CFC SABA inhalers with more costly non-generic hydrofluoroalkane (HFA) inhalers and has increased the economic burden associated with overreliance on SABAs. In 2004, the average cost of a HFA albuterol MDI across all payer types was 2.9 times greater than a generic CFC albuterol MDI (\$39.50 vs \$13.50, respectively). ${ }^{5}$

One key element in achieving asthma control and thereby reducing healthcare utilization and costs, is proper adherence to prescribed asthma treatment. ${ }^{1}$ Nonadherence to medical treatment regimens is estimated to cost the US healthcare system $\$ 100$ billion annually. ${ }^{6}$ Among patients with asthma, reduced adherence to prescribed medication has been associated with the need for increased medical care and reduced asthma control, including increased ED visits, increased OCS use, increased exacerbations, and/or worse asthma symptom scores. ${ }^{7-10}$ Because current NAEPP guidelines indicate that low-dose inhaled corticosteroids (ICSs) are the recommended treatment for mild persistent asthma, ${ }^{1}$ ICS monotherapy is the most important type of treatment to consider for assessing the association between adherence and mild persistent asthma control. Factors that may affect adherence for patients with mild persistent asthma include ease and frequency of ICS administration and patients' perceptions of treatment effectiveness. Previous data indicate that patients with asthma are more likely to be highly adherent to once-daily ICS therapy compared with twice-daily ICS therapy. ${ }^{11}$ However, adequate formal analyses of the difference in charges and resource utilization of ICS treatment between highly adherent and minimally adherent patients with mild asthma are lacking from the current biomedical literature.

The primary objective of the current study was to determine the impact of adherence and asthma control on resource use and asthma treatment charges in patients with mild asthma having ICS claims. A secondary objective was to determine which ICS treatment was associated with the greatest likelihood of achieving high control and high adherence, which may indicate which ICS therapies are best for optimizing asthma control.

\section{Patients and methods Study design}

A claims database (Ingenix LabRx, Eden Prairie, MN, USA) was analyzed retrospectively from October 2001 through December 2007 to identify patients with mild asthma who began treatment with an ICS, including but not limited to mometasone furoate (MF; Asmanex ${ }^{\circledR}$, Merck \& Co., Inc., Whitehouse Station, NJ, USA), fluticasone propionate (FP; Flovent $^{\circledR}$, GlaxoSmithKline, Research Triangle Park, NC, USA), FP HFA (Flovent ${ }^{\circledR}$ HFA, GlaxoSmithKline, Research Triangle Park, NC, USA), budesonide (Pulmicort ${ }^{\circledR}$, AstraZeneca LP, Wilmington, DE, USA), or beclomethasone dipropionate (Qvar ${ }^{\circledR}$, 3M Drug Delivery Systems, Northridge, CA, USA). During the timeframe used for this analysis, the database included approximately 37 million patients who primarily resided in the Southern or Midwestern United States (South, 43\%; Midwest, 33\%; West, 13\%; Northeast, $11 \%$ ). For each patient, the date of the first ICS prescription fill was considered the index date. Demographic information, drug utilization, and resource use for each patient were identified for the 365-day period before the index date (pre-index period) and the 365-day period following the index date (postindex period). Information collected during the pre-index period included total number of SABA canister prescriptions filled; incidence of comorbidities, including pneumonia, sinusitis, acute bronchitis, acute laryngitis, upper respiratory infection, acute nasopharyngitis, gastroesophageal reflux disease, and rhinitis; total asthma-related charges; pre-index asthma days; and pre-index asthma records. Asthma days was defined as the number of distinct days in which the patient had a medical record with any diagnosis of asthma in the pre-index period. Pre-index asthma records was defined as the number of distinct asthma-related records, identified by the presence of an asthma-related International Classification of Diseases, 9th Edition, Clinical Modification (ICD-9-CM) code in which the patient had a medical record with a diagnosis of asthma in the pre-index period; note that a single office visit may have resulted in $>1$ asthma record.

\section{Patients}

All patients met the following inclusion/exclusion criteria: received an ICS prescription between October 2001 and 
December 2007; enrolled in health plans at least 1 year before and 1 year after index date; were 12 to 65 years of age; had no other chronic pulmonary condition (eg, identified by ICD-9-CM codes 415X, 416X, 417X, 491X, 491.2X, 492X, 493.2X, 494X, or 770.2X); received no combination therapy within 7 days of the index date; did not switch asthma therapy during post-index period; were designated as having mild asthma using an algorithm developed previously; ${ }^{12}$ and were designated as having high control, high adherence (HCHA) or low control, low adherence (LCLA). Patients with mild asthma were defined as those who had been diagnosed with an ICD-9-CM code of 493.0X, 493.1X, or 493.9X and did not experience an asthma exacerbation or use $>2$ SABA canisters during the 365-day pre-index period. Although these criteria for mild asthma are not clinically based, similar methods have been used in other analyses. ${ }^{12-14}$ An asthma exacerbation was defined as an asthma episode that required hospitalization, treatment in an emergency room, or an outpatient visit during which nebulization or a prescription for OCSs was given, as previously described. ${ }^{12}$ For adherence, the percent days covered (PDC) was defined as the percentage of days that patients had access to medication assuming daily drug use. The HCHA cohort included patients with no exacerbation events (high control) and $\geq 60 \%$ PDC (high adherence) during the post-index period. The LCLA cohort included patients with $\geq 2$ exacerbation events (low control) and $<10 \%$ PDC (low adherence) during the post-index period. Low adherence was selected as $<10 \%$ PDC because this corresponds with patients who generally had 1 and only 1 prescription (eg, a median 30-day supply) during the 1-year post-index period. These cutoffs were based on distributions of exacerbations and PDC using upper and lower quartiles (eg, no priori determinations were made regarding patient cutoff points for designation as HCHA or LCLA).

\section{Assessments}

The primary outcomes of this analysis were post-index comparisons between the HCHA and LCLA cohorts in asthma-related resource use (eg, asthma-related medical records, asthma treatment days, and OCS claims) and charges (eg, outpatient charges, inpatient charges, pharmaceutical charges, and total asthma charges). All asthma charges were adjusted to 2008 US dollars.

Secondary outcomes of this analysis included the percentage of patients in each ICS treatment group who belonged in the HCHA cohort and odds ratios for the likelihood of belonging to the HCHA cohort based on ICS treatment initiated on the index date. This analysis was limited to ICSs for which patient sample sizes in the database were $\geq 500$.

\section{Statistical analyses}

Bivariate analyses of the HCHA and LCLA cohorts were performed to determine differences in asthma resource use and overall asthma treatment charges. A 2-by-2 contingency table chi-square analysis was preformed to compare the unadjusted odds of patients belonging to the HCHA cohort based on ICS treatment. A forward stepwise logistic regression model was built to evaluate the odds ratio of a patient on a specific ICS therapy belonging to the HCHA cohort, adjusting for other covariates in the model.

\section{Results}

A total of 741 patients were included in the current analysis (Figure 1). Because this analysis was designed to compare patients at the extreme ends of the adherence/control spectrum, only $4 \%$ of patients who met all other inclusion/ exclusion criteria qualified for either the HCHA or LCLA cohort (Figure 1). The majority of patients were female; the mean age of patients was 44.2 and 33.4 years in the HCHA and LCLA cohorts, respectively (Table 1). The HCHA cohort had a higher age, a lower proportion of female patients, a larger number of asthma days and asthma-related records, and higher asthma-related charges during the pre-index period $(P<0.0001$; Table 1$)$. Both cohorts were similar in the proportions of patients with measured comorbidities, with the exception of rhinitis (higher in the HCHA cohort $[P<0.0001$; Table 1]) and upper respiratory tract infection (higher in the LCLA cohort $[P<0.0001$; Table 1]).

\section{Resource use and treatment charges: bivariate analyses}

Compared with the HCHA cohort, the LCLA cohort was associated with more mean asthma-related medical records (5.6 vs 9.7, respectively; $P<0.0001$ ), more mean asthma treatment days ( 2.9 vs 3.9 , respectively $P<0.0001)$, more mean OCS prescriptions ( 0.0 vs 0.4 , respectively; $P<0.0001$ ), and fewer mean ICS records (7.7 vs 1.0, respectively; $P<0.0001$ ) in the post-index period (Figure 2).

The HCHA cohort was associated with lower total mean asthma charges in the post-index period compared with the LCLA cohort (\$2655 vs \$3345, respectively, $P<0.0001$; Figure 3). Mean pharmaceutical asthma charges were significantly higher for the HCHA cohort compared with the LCLA cohort ( $\$ 1085$ vs $\$ 129$, respectively, $P<0.0001$; Figure 3), as one would expect based on the cohort definitions. 
The lower overall mean asthma treatment charges in the HCHA cohort were primarily driven by lower resource use (ie, inpatient/outpatient charges) compared with the LCLA cohort (inpatient charge, $\$ 19$ vs $\$ 1248$, respectively, $P<0.0001$; outpatient charge, $\$ 1551$ vs $\$ 1968, P=0.0001$; Figure 3).

\section{ICS use associated with $\mathrm{HCHA}$ : multivariate analyses}

Among patients who met all inclusion criteria and received an ICS that was prescribed to $\geq 500$ patients in the database $(n=16,521)$, a total of 450 patients belonged to the HCHA cohort. A significantly higher percentage of patients in this subanalysis who received MF belonged in the HCHA

Inclusion/Exclusion
Asthma patients with an
ICS prescription identified
Enrolled $\geq 1$ y before and
$\geq 1$ y after index date
12-65 y of age
No other chronic
pulmonary condition
No combination therapy
within 7 d of index date
Did not receive treatment with the
therapy of another treatment cohort
within 7 days of index date*
Belonged to only 1
treatment cohort*
Did not switch asthma therapy
during postindex period
mild asthma

Figure I Patient selection. Patients were identified from a commercial insurance database and analyzed retrospectively to identify those with mild asthma who initiated treatment with an ICS. Patients who met all inclusion and exclusion criteria were assigned to I of 2 control/adherence cohorts based on the number of exacerbation events and percent days covered in the pre-index period: $\mathrm{HCHA}, 0$ exacerbation events and $\geq 60 \%$ PDC; LCLA, $\geq 2$ exacerbation events and $<10 \%$ PDC.

Abbreviations: ICS, inhaled corticosteroid; $\mathrm{HCHA}$, high control high adherence; LCLA, low control low adherence; PDC, percent days covered.

*Criterion included owing to the initial existence of a fluticasone propionate with salmeterol cohort, which is not presented in the current analysis. cohort than any other ICS $(P<0.0001$; Figure 4). Using an unadjusted chi-square analysis, MF patients were 7.425 times more likely than patients receiving other ICS agents to be in the HCHA cohort $(P<0.0001)$. Calculation of odds ratios from the logistic regression model revealed that patients receiving MF were 5.081 (95\% CI, 4.144-6.230) times more likely than patients receiving other ICS agents to be in the HCHA cohort $(P<0.0001)$ after adjusting for all other pre-index variables.

\section{Discussion}

The results of the current analysis suggest that increases in asthma control and adherence to prescribed asthma medication correlate with decreases in total asthma-related charges. Among patients with a low level of asthma control, who were minimally adherent to their prescribed ICS medication, the incidence of asthma-related records, asthma treatment days, and the number of OCS prescriptions were higher compared with patients who had a superior level of asthma control and were more adherent to their prescribed ICS medication. This finding is not surprising given the strong correlation between asthma patient adherence to prescribed medication and asthma control, ${ }^{7-10}$ and the increased costs associated with asthma exacerbations ${ }^{15}$ and/or extra doctor visits ${ }^{16}$ that often accompany poorly controlled asthma. In a large retrospective analysis of a managed care database by Stern and colleagues $(\mathrm{N}=97,743)$, patients who were more highly compliant to their prescribed controller medication (including ICSs) were significantly less likely to experience an asthma-related exacerbation $(P<0.001) .{ }^{9}$ Other studies

Table I Demographics and characteristics at index date

\begin{tabular}{llll}
\hline $\begin{array}{l}\text { Demographic or } \\
\text { characteristic }\end{array}$ & $\begin{array}{l}\text { HCHA } \\
(\mathbf{n}=\mathbf{4 8 3})\end{array}$ & $\begin{array}{l}\text { LCLA } \\
(\mathbf{n}=\mathbf{2 5 8})\end{array}$ & P value \\
\hline Mean age, $\mathrm{y}$ & 44.2 & 33.4 & $<0.0001$ \\
Female, \% & 61.5 & 76.4 & $<0.000 \mathrm{I}$ \\
Mean SABA claims, $\mathrm{n}$ & 0.50 & 0.56 & 0.1844 \\
Asthma days, $\mathrm{n}$ & 1.7 & 1.0 & $<0.000 \mathrm{I}$ \\
Pre-asthma records, $\mathrm{n}$ & 3.7 & 1.8 & $<0.000 \mathrm{I}$ \\
Mean asthma-related charges, $\$$ & 502 & 297 & $<0.000 \mathrm{I}$ \\
Comorbidity, \% & & & \\
$\quad$ Rhinitis & 48.0 & 30.2 & $<0.000 \mathrm{I}$ \\
Sinusitis & 27.5 & 29.8 & 0.5065 \\
$\quad$ Upper respiratory infection & 15.5 & 31.4 & $<0.000 \mathrm{I}$ \\
Acute bronchitis & 15.5 & 18.6 & 0.2836 \\
GERD & 14.1 & 11.2 & 0.2752 \\
Pneumonia & 2.3 & 4.3 & $0.129 \mathrm{I}$ \\
Acute laryngitis & 1.7 & 2.3 & 0.5238 \\
Acute nasopharyngitis & 1.9 & 1.6 & 0.7572 \\
\hline
\end{tabular}

Abbreviations: GERD, gastroesophageal reflux disease; $\mathrm{HCHA}$, high control high adherence; LCLA, low control low adherence; SABA, short-acting $\beta_{2}$-agonist. 


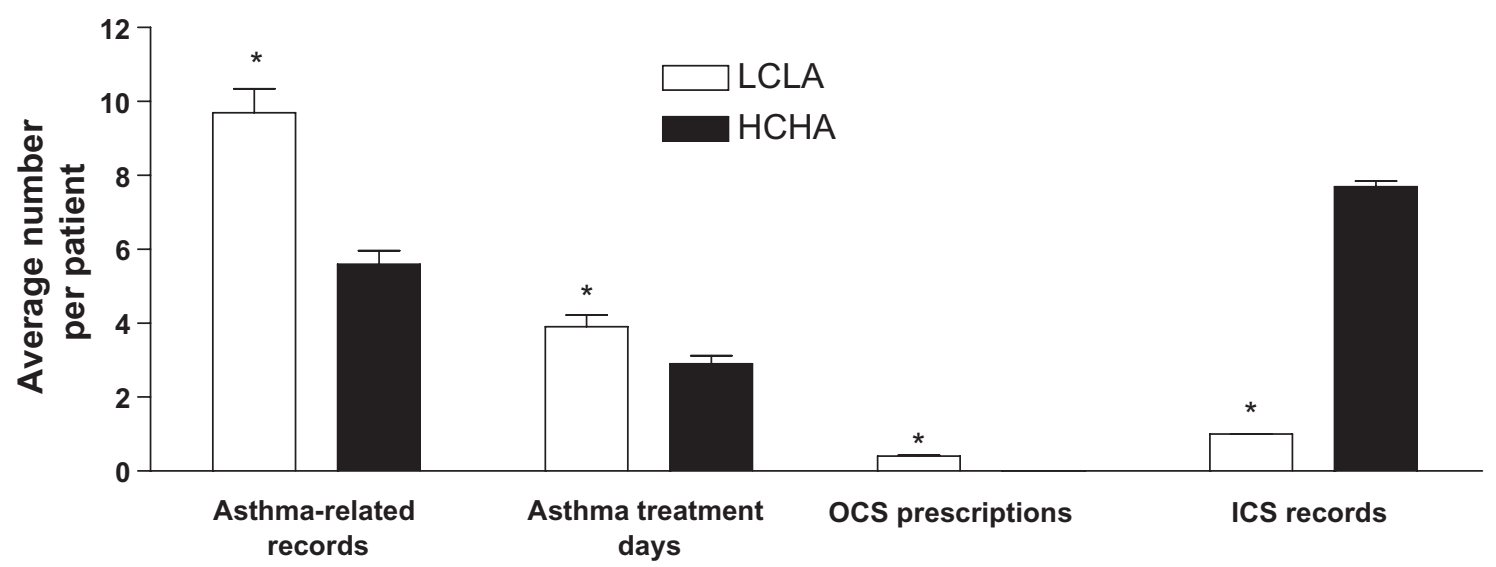

Patient record type

Figure 2 Resource utilization. The mean number of post-index asthma-related medical records, asthma days, OCS prescriptions, and ICS records among patients assigned to the HCHA or LCLA cohort are depicted. Error bars represent standard error of the mean.

Abbreviations: $\mathrm{HCHA}$, high control high adherence; ICS, inhaled corticosteroids; LCLA, low control low adherence; OCS, oral corticosteroids. $* p<0.000$ I.

that specifically assessed adherence to ICS treatment have found that enhanced patient adherence is associated with increased asthma control as measured by overall asthma control scores, ${ }^{7,8}$ emergency department visits, ${ }^{10}$ and OCS prescriptions. ${ }^{10}$ In a prospective cohort study by Krishnan and colleagues $(\mathrm{N}=60)$, asthma patients with poor adherence had significantly worse asthma symptom scores $(P=0.04) 2$ weeks after hospital discharge following an asthma exacerbation. ${ }^{7}$ In a large, descriptive, observational study by Molimard and Le Gros $(\mathrm{N}=4362)$, asthma control was deemed inadequate among $62.7 \%$ of persistent asthma patients who missed $\geq 4$ ICS doses per week, but only
$37.5 \%$ of patients who missed $<4$ ICS doses per week. ${ }^{8}$ In a retrospective claims database analysis of asthma patients by Williams and colleagues $(\mathrm{N}=405)$, ICS adherence correlated negatively and significantly to emergency department visits and OCS prescriptions; furthermore, each $25 \%$ increase in the percentage of time that patients were without ICS therapy doubled the asthma-related hospitalization rate. ${ }^{10}$

The lower asthma-related days and records observed in the HCHA cohort compared with the LCLA cohort is in contrast to the index date data, when these parameters were higher in the HCHA cohort. This may suggest subjects in the HCHA cohort had slightly more severe (although still mild)

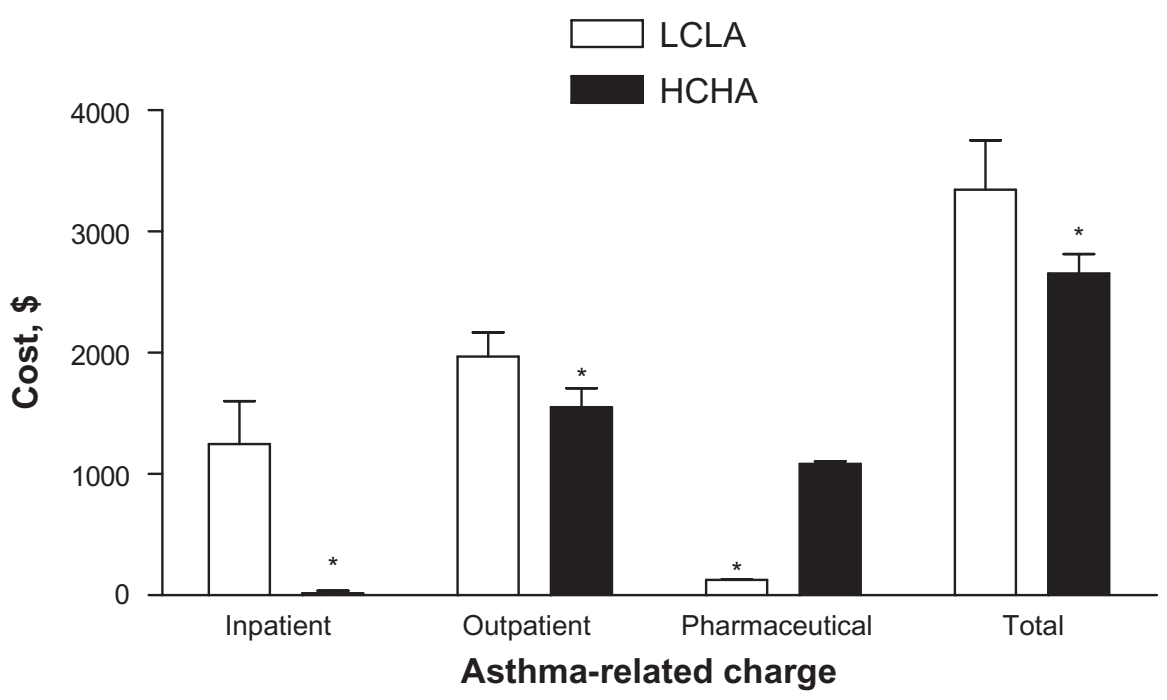

Figure 3 Asthma-related charges. The mean post-index asthma-related inpatient, outpatient, pharmaceutical, and total charges among patients assigned to the HCHA or LCLA cohort are depicted. Error bars represent standard error of the mean.

Abbreviations: HCHA, high control high adherence; LCLA, low control low adherence. $* P \leq 0.0001$. 


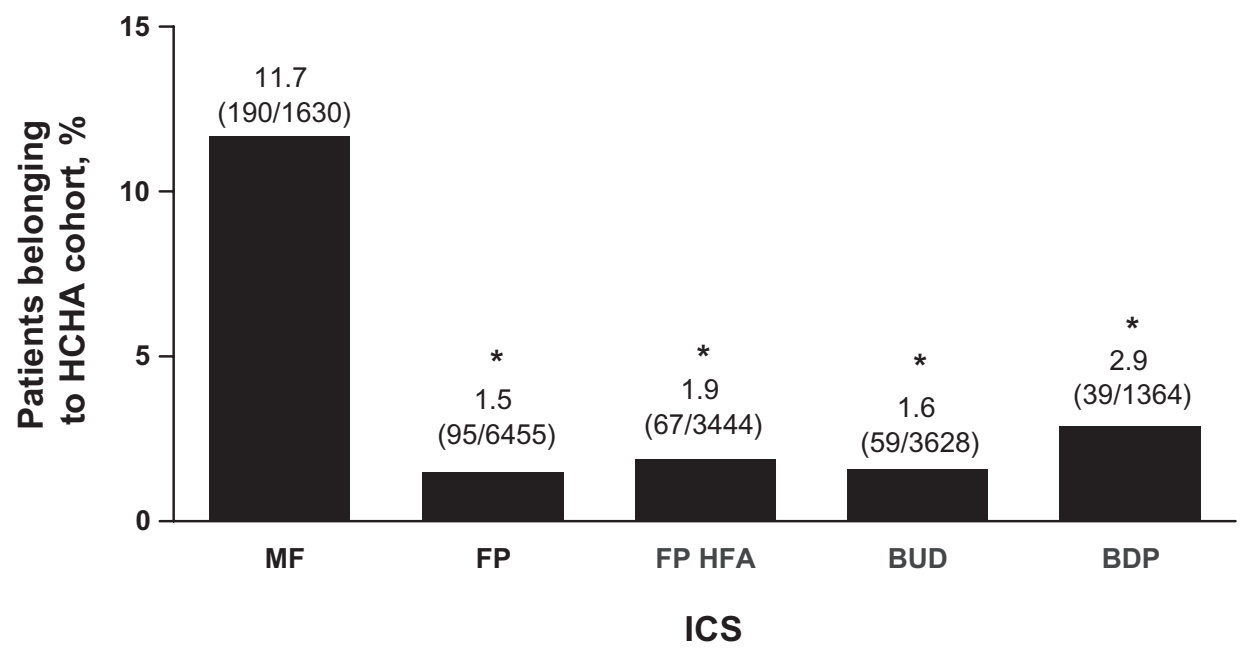

Figure 4 ICS use associated with HCHA. The percentages of patients included in the current analysis that were treated with MF, FP, FP HFA, BUD, or BDP and assigned to the HCHA cohort are depicted. Percentages for each ICS group represent the number of patients who were treated with each respective ICS and qualified for the HCHA cohort divided by the total number of patients who were treated with each respective ICS. Error bars represent standard error of the mean.

Abbreviations: BDP, beclomethasone dipropionate; BUD, budesonide; FP, fluticasone propionate; HCHA, high control high adherence; HFA, hydrofluoroalkane; ICS, inhaled corticosteroid; LCLA, low control low adherence; MF, mometasone furoate.

$* P<0.0001$ vs MF.

asthma resulting in more asthma-related visits. The resulting decrease in asthma-records and days during the post-index period may be due to implementation of ICS treatment resulting in control (prior ICS use was not part of the inclusion criteria), a switch to more effective therapy, or an emphasis on drug adherence by the prescribing physician.

Collectively, these data demonstrate that enhanced adherence to prescribed ICS therapy correlates with improved asthma control. As such, it is critical to optimize patient adherence to not only improve patient outcomes, but also to lower the global economic burden of asthma care. The increased asthma-related exacerbations, emergency room visits, and inpatient and outpatient doctor visits associated with poorly controlled asthma result in significant pharmacoeconomic burden. ${ }^{15,16} \mathrm{~A}$ recent retrospective analysis of the 2004 Medical Expenditure Panel Survey data by Kamble and Bharmal estimated that asthma afflicts 6.4 million children and 14.8 million adults in the United States alone and costs approximately $\$ 1000$ and $\$ 2000$, respectively, per person annually in 2007 US dollars. ${ }^{16}$ All medical visits combined (including inpatient, outpatient, emergency room, and office-based medical visits) accounted for approximately $60 \%$ of all asthma-related expenditures in children and approximately $40 \%$ of all asthma-related expenditures in adults. Given the significant overall economic burden of asthma and the association between asthma control and patient adherence to prescribed medication, improvements in patient adherence and asthma control have the potential to significantly reduce asthma-related healthcare expenditures.
As expected, data from the current study suggested that reduced asthma control and adherence to prescribed asthma medication correlated with increased asthma-related total charges driven by increased inpatient and outpatient charges. Previous studies have demonstrated similar correlations between asthma control, adherence, and resource use and charges. Among asthma patients $(\mathrm{N}=527)$ stratified to 1 of 4 quartiles of asthma control that ranged from "good control" to "poor control," Accordini and colleagues found that hospitalizations and doctor visits increased significantly with decreasing levels of controller medication use and poor asthma control, and generally correlated with higher asthma-related direct medical expenditures and indirect costs. ${ }^{3}$ For all 5 mean annual cost parameters assessed (ie, doctor visits and lab visits, pharmacological treatment, hospitalization, indirect costs, and total), the estimated mean annual cost per patient increased as the level of disease control decreased $(P<0.001) .{ }^{3}$ In parallel with results from the current study, patients with poor asthma control experienced the lowest percentage of direct charges due to pharmacological treatment and the highest percentage of direct charges due to hospitalization, whereas patients with good asthma control experienced notably higher pharmacological treatment charges and lower direct charges. ${ }^{3}$ Collectively, these data suggest that the total expenses associated with asthma-related charges are lower for patients with superior asthma control, driven by reductions in hospital and physician-related services (ie, inpatient and outpatient visits, hospitalizations, and ED visits) charges, but not reductions 
in prescription medication charges. This finding is somewhat surprising because the greatest contributor to asthma care charges in the United States is prescription drugs, which are estimated to account for $43 \%$ of the $\$ 16.1$ billion spent on asthma care annually. ${ }^{17}$ However, it is possible that minimally adherent patients with mild persistent asthma who have poorly controlled asthma have lower pharmaceutical asthmarelated charges because they do not adhere to their prescribed dosing regimens of controller medications, such as ICSs. As such, these patients may use less of their prescribed medication, which may lead to complications that result in asthma exacerbations, hospitalizations, ER visits, and/or more frequent trips to see physicians. The current data suggest that highly adherent asthma patients who achieve a high level of asthma control may have more charges for prescription medication than patients with a lower level of adherence and asthma control, but the tradeoff for this higher expense is fewer inpatient and outpatient physician visits, which corresponds with lower total medical care charges.

There are several reasons why patients who received MF were approximately 5 times more likely to be in the HCHA cohort than patients who received the other ICSs. In general, there are limited data that suggest clinically relevant differences between ICSs in clinical efficacy. ${ }^{18}$ However, study outcomes that may be related to patient adherence (ie, asthma control) will tend to be minimal in well organized clinical trials because such trials are often designed to limit differences between treatment groups in adherence. The oncedaily dosing regimen of MF may be the most likely reason why a higher percentage of patients receiving MF were in the HCHA cohort compared with patients who received one of the other ICSs. Previous data suggest that adherence to prescribed medication, regardless of disease state, increases as daily doses decrease. ${ }^{19}$ Guest et al specifically investigated the change in adherence when patients with asthma were switched from a twice-daily ICS to once-daily ICS treatment. ${ }^{11}$ As might be expected, patients who switched to a once-daily ICS were more likely to be highly adherent and had lower asthma-related charges compared with patients who switched to another twice-daily ICS dosing regimen. ${ }^{11}$ In addition, Price et al found that for MF specifically, adherence was significantly higher for once-daily dosing than twicedaily dosing. ${ }^{20}$ NAEPP guidelines note the importance of assessing and encouraging asthma patient adherence to prescribed therapy, ${ }^{1}$ and point out that adherence to a therapeutic plan is enhanced when daily doses are minimized. ${ }^{21-23}$ Further analyses in larger sample sizes of patients are necessary to verify if MF treatment is associated with lower asthma-related charges among highly adherent, high asthma control patients compared with other ICSs because of a superior dosing regimen. Furthermore, it is important to note that although most patients with asthma treated with MF are prescribed a once-daily dosing regimen, some patients (ie, those $\geq 12$ years of age receiving oral OCSs) may receive a twice-daily regimen, ${ }^{24}$ and it is impossible to determine how many patients were prescribed once-daily MF versus twice-daily MF in the current claims database analysis. However, because of their designation as mild asthma patients, it is likely that the majority of patients in the current study who received MF were prescribed a once-daily regimen.

An alternative or additional explanation for the increased adherence with MF patients is that the MF device (Twisthaler ${ }^{\circledR}$, Merck \& Co., Inc., Whitehouse Station, NJ, USA) is easy to use. There are only 3 steps in the dosing process and the device has an integrated dose counter. ${ }^{25}$ In a head-to-head comparison of the MF Twisthaler and a fluticasone propionate metered-dose inhaler, there was no significant difference in the number of subjects who rated the inhalers "easy to use," but significantly more subjects using Twisthaler "liked it a lot" ( $47 \%$ vs $22 \%, P=0.01) .{ }^{26}$ Ease of use and patient satisfaction with an inhaler may contribute to adherence, but this area of research has not been adequately explored.

One limitation of the overall design of the current study was that it only included patients with a low level of asthma control and adherence to their prescribed ICS medication, or a high level of asthma control and adherence to their prescribed ICS medication. In real-world clinical settings, many asthma patients fall somewhere in between this continuum, with "medium" levels of both asthma control and adherence to therapy. Previous data suggest that, regardless of asthma severity, improved asthma control is associated with lower asthma-related healthcare costs ${ }^{3}$ and higher patient adherence to prescribed therapy. ${ }^{7-10}$ However, specific analyses of patients with a low level of asthma control and good adherence to prescribed therapy, or a low level of asthma control and poor adherence to prescribed therapy are lacking from the biomedical literature. One reason for this gap in the literature may be because it is rare to find patients who are not misdiagnosed who have unparalleled levels of asthma control and adherence to therapy. For all patients, physicians should promote adherence to prescribed treatment in accordance with current NAEPP guidelines ${ }^{1}$ to optimize each individual patient's respective asthma control potential and minimize healthcare costs. For patients with a low level of asthma control who are highly adherent to their prescribed therapy, it may be necessary for physicians to re-evaluate the 
treatment plan being employed to determine if alternate therapeutic options should be pursued.

There are several limitations to this type of observational claims database analysis. The retrospective nature of the study limited the amount and type of information that could be collected, such as information about why a specific ICS was chosen or how adherence could be measured. Also, it is difficult to accurately categorize asthma severity without using predefined clinical outcome measures; ${ }^{13}$ one previous claims-based analysis categorized patients as having mild asthma less frequently than did pulmonary function testing in the same population. ${ }^{14}$ In addition, the definitions of HCHA and LCLA used in this analysis were not standardized and it is difficult to verify the accuracy of data found in a claims database. However, these limitations are inherent to any claims database analysis and do not preclude the development of important and clinically relevant conclusions about the effect of asthma control and adherence on asthma-related charges and resource use.

\section{Conclusions}

Collectively, these data suggest that better asthma control and adherence to prescribed ICSs are associated with lower overall asthma-related resource use and charges. Furthermore, patients with mild asthma receiving MF were more likely to be in the HCHA patient cohort than patients receiving a different $I C S$, which may have been due to the once-daily dosing regimen of MF. Of critical importance to optimizing mild persistent asthma control and limiting asthma-related resource use and charges is following current NAEPP guidelines, which indicate low-dose ICS monotherapy as the preferred treatment for mild persistent asthma. $^{1}$

\section{Transparency \\ Declaration of funding}

This work was supported by Schering-Plough Corporation, now Merck \& Co., Inc.

\section{Declaration of financial/other relationships}

Prakash Navaratnam is a paid consultant for Merck \& Co., Inc.

Howard S Friedman is the owner of Analytic Consulting, LLC which performs consulting work in the pharmaceutical industry, including Merck \& Co., Inc.

Eduardo Urdaneta was an employee of Schering-Plough Corporation when the study was conducted.

\section{Acknowledgments}

Medical writing assistance was provided by Brett Mahon, $\mathrm{PhD}$ and was funded by Schering-Plough Corporation, now Merck \& Co., Inc. Additional assistance was provided by John McLaughlin, MSPH.

\section{References}

1. National Asthma Education and Prevention Program. Expert Panel Report 3: Guidelines for the Diagnosis and Management of Asthma 2007. Available at: http://www.nhlbi.nih.gov/guidelines/asthma/index. htm [Last accessed April 2008].

2. Masoli M, Fabian D, Holt S, Beasley R. The global burden of asthma: executive summary of the GINA Dissemination Committee report. Allergy. 2004;59:469-478.

3. Accordini S, Bugiani M, Arossa W, et al. Poor control increases the economic cost of asthma. A multicentre population-based study. Int Arch Allergy Immunol. 2006;141:189-198.

4. Briggs AH, Bousquet J, Wallace MV, et al. Cost-effectiveness of asthma control: an economic appraisal of the GOAL study. Allergy. 2006;61:531-536.

5. Food and Drug Administration. Use of ozone-depleting substances; removal of essential-use designations; final rule. Fed Regist. 2005;70:17168-17192.

6. Horne R. Compliance, adherence, and concordance: implications for asthma treatment. Chest. 2006;130:65S-72S.

7. Krishnan JA, Riekert KA, McCoy JV, et al. Corticosteroid use after hospital discharge among high-risk adults with asthma. Am J Respir Crit Care Med. 2004;170:1281-1285.

8. Molimard M, Le Gros V. Impact of patient-related factors on asthma control. J Asthma. 2008;45:109-113.

9. Stern L, Berman J, Lumry W, et al. Medication compliance and disease exacerbation in patients with asthma: a retrospective study of managed care data. Ann Allergy Asthma Immunol. 2006;97:402-408.

10. Williams LK, Pladevall M, Xi H, et al. Relationship between adherence to inhaled corticosteroids and poor outcomes among adults with asthma. J Allergy Clin Immunol. 2004;114:1288-1293.

11. Guest JF, Davie AM, Ruiz FJ, Greener MJ. Switching asthma patients to a once-daily inhaled steroid improves compliance and reduces healthcare costs. Prim Care Respir J. 2005;14:88-98.

12. Friedman HS, Yawn BP. Resource utilization in asthma: combined fluticasone propionate/salmeterol compared with inhaled corticosteroids. Curr Med Res Opin. 2007;23:427-434.

13. Colice G, Wu EQ, Birnbaum H, et al. Healthcare and workloss costs associated with patients with persistent asthma in a privately insured population. J Occup Environ Med. 2006;48:794-802.

14. Birnbaum HG, Ivanova JI, Yu AP, et al. Asthma severity categorization using a claims-based algorithm or pulmonary function testing. J Asthma. 2009;46:67-72.

15. Lane S, Molina J, Plusa T. An international observational prospective study to determine the cost of asthma exacerbations (COAX). Respir Med. 2006;100:434-450.

16. Kamble $\mathrm{S}$, Bharmal M. Incremental direct expenditure of treating asthma in the United States. J Asthma. 2009;46:73-80.

17. American Lung Association. Trends in Asthma Morbidity and Mortality. New York, NY; Epidemiology and Statistics Unit. New York, NY.

18. Barnes NC. The properties of inhaled corticosteroids: similarities and differences. Prim Care Respir J. 2007;16:149-154.

19. Claxton AJ, Cramer J, Pierce C. A systematic review of the associations between dose regimens and medication compliance. Clin Ther. 2001;23:1296-1310.

20. Price D, Robertson A, Bullen K, et al. Improved adherence with once-daily versus twice-daily dosing of mometasone furoate administered via a dry powder inhaler: a randomized open-label study. BMC Pulm Med. 10:1. 
21. Bender BG, Bender SE. Patient-identified barriers to asthma treatment adherence: responses to interviews, focus groups, and questionnaires. Immunol Allergy Clin North Am. 2005;25:107-130.

22. Eisen SA, Miller DK, Woodward RS, et al. The effect of prescribed daily dose frequency on patient medication compliance. Arch Intern Med. 1990;150:1881-1884.

23. Haynes RB, Yao X, Degani A, et al. Interventions to enhance medication adherence. Cochrane Database Syst Rev. 2005;CD000011.

24. Asmanex ${ }^{\circledR}$ Twisthaler $^{\circledR}$ (mometasone furoate). Full Prescribing Information, Merck \& Co., Inc., Whitehouse Station, NJ, USA, 2008.
25. Karpel JP. An easy-to-use dry-powder inhaler. Adv Ther. 2000; 17:282-286.

26. Wardlaw A, Larivee P, Eller J, et al. Efficacy and safety of mometasone furoate dry powder inhaler vs fluticasone propionate metered-dose inhaler in asthma subjects previously using fluticasone propionate. Ann Allergy Asthma Immunol. 2004;93:49-55.

\section{Publish your work in this journal}

Patient Preference and Adherence is an international, peer-reviewed, open access journal that focusing on the growing importance of patient preference and adherence throughout the therapeutic continuum. Patient satisfaction, acceptability, quality of life, compliance, persistence and their role in developing new therapeutic modalities and compounds to optimize clinical outcomes for existing disease states are major areas of interest. This journal has been accepted for indexing on PubMed Central. The manuscript management system is completely online and includes a very quick and fair peer-review system. Visit http://www.dovepress.com/ testimonials.php to read real quotes from published authors.

Submit your manuscript here: http://www.dovepress.com/patient-preference-and-adherence-journal 